\title{
Transcription factor NFAT5 contributes to the glycolytic phenotype rewiring and pancreatic cancer progression via transcription of PGK1
}

Yongsheng Jiang ${ }^{1,2}$, Ruizhe He${ }^{1}$, Yuhong Jiang ${ }^{3}$, Dejun Liu', Lingye Tao ${ }^{1}$, Minwei Yang ${ }^{1}$, Chaoyi Lin ${ }^{1}$, Yang Shen ${ }^{1}$, Xueliang Fu (1)', Jianyu Yang ${ }^{1}$, Jiao Li ${ }^{4}$, Yanmiao Huo ${ }^{1}$, Rong Hua ${ }^{1}$, Wei Liu', Junfeng Zhang ${ }^{1}$, Baiyong Shen², Zhigang Zhang (1) and Yongwei Sun (1)

\begin{abstract}
Hypoxia and the hypovascular tumor microenvironment are major hallmarks of pancreatic ductal adenocarcinoma (PDAC), in which glycolysis is of great importance to tumor survival and proliferation. There is little research regarding the role of Nuclear Factor of Activated T Cells 5 (NFAT5) in relation to carcinoma. Here, we explored the impact of NFAT5 on the biological behavior of PDAC and the underlying mechanism. We demonstrated that NFAT5 was highly expressed in PDAC and was related to poorer prognosis. Knockdown of NFAT5 lead to impaired proliferation of tumor cells caused by an aberrant Warburg effect. Mechanically, phosphoglycerate kinase 1 (PGK-1), which is the first enzyme generating ATP in glycolysis, was verified as a target gene of NFAT5. Over-expression of PGK1 compromised the aberrant oncological behavior caused by knockdown of NFAT5 both in vitro and in vivo. Clinical samples underwent positron emission tomography-computed tomography (PET-CT) examination and KrasG12D/+/Trp53R172H/+/Pdx1Cre (KPC) mice were collected to support our conclusion.
\end{abstract}

\section{Introduction}

Pancreatic cancer is one of the most aggressive and lethal malignant tumors, with a continuously rising mortality ${ }^{1,2}$. Pancreatic cancer ranks fourth among the fatal malignant tumors in western countries and sixth in China; its 5 -year survival rate is only $7 \%$ or lower $^{2-4}$. Because of its unique anatomical location and structure, as well as its physiological characteristics, it has no obvious symptoms at the early stage of onset and it is consequently quite difficult to diagnose. For the purpose

Correspondence: Baiyong Shen (shenby@shsmu.edu.cn) or

Zhigang Zhang (zzhang@shsci.org) or Yongwei Sun (syw0616@126.com)

${ }^{1}$ Department of Biliary-Pancreatic Surgery, Ren Ji Hospital, School of Medicine,

Shanghai Jiao Tong University, Shanghai, P. R. China

${ }^{2}$ Department of General Surgery, Rui Jin Hospital, School of Medicine,

Shanghai Jiao Tong University, Shanghai, P. R. China

Full list of author information is available at the end of the article.

These authors contributed equally: Yongsheng Jiang, Ruizhe He, Yuhong Jiang, Dejun Liu

Edited by I. Amelio of enhancing the survival rate and improving the life quality of patients, biological and genetic studies of pancreatic cancer are imperative.

There is increasing evidence of the pro-tumor role of the NFAT (Nuclear Factor of Activated T cell) family in multiple kinds of malignancies. The NFAT family consists of five transcription factors including NFATc1, NFATc2, NFATc3, NFATc4, and NFAT $5^{5}$, most of which have been well-studied in development processes. For instance, NFATc1 is able to transform preadipocyte cells via the JAK-Stat3 pathway, and NFATc3 can contribute to malignant melanoma progression. However, the specific role of these transcription factors in pancreatic ductal adenocarcinoma (PDAC) has not been well-studied. Here, we found that all five transcription factors are highly expressed in tumor tissue, and the expression of NFAT5 is negatively associated with prognosis. NFAT5 (Nuclear Factor Of Activated T Cells 5) was first recognized as a tonicity-regulated transcription factor (Ton/EBP) that 
regulates the expression of genes involved in osmotic stress $^{6,7}$. Recent studies revealed the potential relationship between NFAT5 and several cancers. In melanoma, NFAT5 was shown to react in the cell cycle and play a driving role in the development and carcinoma metastasis $^{8}$. However, in hepatocellular carcinoma (HCC), NFAT5 was identified as a tumor suppressor gene ${ }^{9,10}$. These findings indicated that NFAT5 has a controversial role in cancer, and the exact role of NFAT5 in pancreatic cancer has not been reported.

In our study, we demonstrated that NFAT 5 contributes to glycolytic phenotype rewiring and pancreatic cancer progression via transcription of PGK1. Glycolysis is active in cancer cells even when oxygen is sufficient. This metabolic characteristic of aerobic glycolysis is known as the Warburg effect ${ }^{11}$. Because pancreatic cancer is a hypovascular tumor ${ }^{12}$, it is essential to investigate the role of the Warburg effect in pancreatic cancer. The Warburg effect has been extensively studied, but its exact essence is still unclear, which hinders the exploration of its therapeutic targets ${ }^{13}$.

Our study demonstrated that NFAT5 is upregulated in pancreatic cancer cells, and NFAT5 facilitates PDAC cell survival via contributing to the Warburg effect by transcription of PGK1.

\section{Materials and methods}

\section{Cell culture and reagents}

Human pancreatic cancer AsPC-1, BxPC-3, CAPAN-1, CFPAC-1, PANC-1, and SW1990 cell lines were purchased from the Cell Bank of the Chinese Academy of Sciences (Shanghai, China). AsPC-1 and BxPC-3 cells were cultured in RPMI 1640 culture medium with $10 \%$ fetal bovine serum (FBS) and 1\% penicillin/streptomycin $(\mathrm{P} / \mathrm{S})$, while the others were cultured in Dulbecco's modified Eagle's medium (DMEM) with $10 \%$ FBS and $1 \% \mathrm{P} / \mathrm{S}$. The culture conditions were $37^{\circ} \mathrm{C}$ and a $5 \%$ $\mathrm{CO}_{2}$ atmosphere. For studies in hypoxia, cells were grown at $37^{\circ} \mathrm{C}$ in an atmosphere of $1 \% \mathrm{O}_{2}$. For the cellular function assay, all PDAC cells were cultured in medium with $5 \mathrm{mM}$ glucose and $2 \mathrm{mM} \mathrm{L}$-glutamine in the absence of FBS. Experiments in Figs. $4 c-f, 5 c$ and $7 \mathrm{~b}-\mathrm{f}$ were performed with cells cultured in hypoxia condition to better simulate the hypovascular tumor microenvironment in PDAC. Cell function assays performed in non-hypoxia conditions were shown in Figs. S1-S3.

\section{Knockdown and overexpression assay}

The lentivirus against NFAT5 was purchased from Gene Pharma (Shanghai, China), and the sequences targeting NFAT5 were: sh-1, 5'-GGCACACAGUCUU GUACAUTT-3' (sense), 5'-AUGUACAAGACUGUGU GCCTT-3' (antisense) and sh-2, 5'-CACUGAGGU
ACCUCGUAA-ATT-3' (sense), 5'-UUUACGAGGUA CCUCAGUGTT-3' (antisense). LV3-pGLV-h1-GFP-puro vector was used for NFAT5 knockdown experiments. For transducing lentivirus, cells were cultured in a six-well plate, and $200 \mu \mathrm{l}$ lentivirus suspension was added in the presence of $5 \mu \mathrm{g} / \mathrm{ml}$ polybrene (Gene Pharma, Shanghai, China). Forty-eight hours after transduction, $5 \mu \mathrm{g} / \mathrm{ml}$ puromycin was added into culture medium for stable cell line screening. For the PGK1 overexpression assay, PGK1expressing plasmid was transfected into the cells in the presence of Lipofectamine 3000 (Thermo Fisher Scientific).

\section{Human PDAC samples, patient information, and tissue microarray (TMA)}

Primary pancreatic cancer tissues were obtained from pancreatic cancer patients treated at Shanghai Jiao Tong University Affiliated Renji Hospital after receiving informed consent. TMA containing 311 pairs of PDAC specimens and corresponding noncancerous tissues were obtained from Ren Ji Hospital from January 2002 to June 2015. For all samples from PDAC patients, clinical information was available. The pathological information was retrieved from the Pathology Department, while the positron emission tomography-computed tomography (PET-CT) images were retrieved from the Nuclear Medicine Department. The follow-up time was calculated from the date of surgery to pancreatic cancer-related death. All the patients were provided with written informed consent before enrolment, and the study was approved by the Research Ethics Committee of Ren Ji Hospital, School of Medicine, Shanghai Jiao Tong University.

\section{Quantitative real-time PCR}

Total RNA was extracted using Trizol reagent (Takara, Japan) according to the manufacturer instructions. cDNA was synthesized using a PrimeScript RT Reagent Kit (Takara, Japan) in accordance with the protocol of the manufacturer. A StepOne Real-Time PCR System (Applied Biosystems, Grand Island, NY, USA) was applied to detect the expression level of the target gene using the SYBR Premix Ex Taq II (Takara, Japan), and $\beta$-actin acted as an internal control. The data were assessed using the 2$\triangle \Delta \mathrm{Ct}$ method. Primer sequences are listed as follows: NFAT5 forward, 5'-GGGTCAAACGACGAGATTG TG-3', reverse, 5'-GTCCGTGGTAAGCTGAGAAAG-3'; GLUT1 forward, 5'-ATTGGCTCCGGTATCGTCAA C-3', reverse, 5'-GCTCAGATAGGACATCCAGGGT A-3'; HK2 forward, 5'-AGCCCTTTCTCCATCTCCT T-3', reverse, 5'-GCTTGCCTACTTCTTCACGG-3'; GPI1 forward, 5'-CAAGGACCGCTTCAACCACTT-3', reverse, 5'-CCAGGATGGGTGTGTTTGA-CC-3'; PFKL forward, 5'-GGTGCCAAAGTCTTCCTCAT-3', reverse, 
5'-GATGATGTTG-GAGACGCTCA-3'; ALDOA forward, 5'-AACTTTCCTCTGCCTAGCCC-3', reverse, 5' GTACAGGCACAGTCGCAGAG-3'; TPI1 forward, 5' -A GCTCATCGGCACTCTGAAC-3', reverse, 5'-CCAC AGCAATCTTGGGATCT-3'; PGK2 forward, 5'-AAA CTGGATGTTAGA-GGGAAGCG-3', reverse, 5'-GGCC GACCTAGATGACTCATAAG-3'; PGAM2 forward, 5'AGAAGCACCCCTACTACAACTC-3', reverse, 5' ${ }^{\prime}$-TCT GGGGAACAA-TCTCCTCGT-3'; ENO1 forward, 5'GCCGTGAACGAGAAGTCCTG-3', reverse, 5'-ACG CCTGAA-GAGACTCGGT-3'; PKM2 forward, 5'-ATGT CGAAGCCCCATAGTGAA-3', reverse, 5'-TGGGTG GTGAATCAATGTCCA-3'; LDHA forward, 5'-ATGG CAACTCTAA-AGGATCAGC-3', reverse, 5' ${ }^{\prime}$-CCA ACCCCAACAACTGTAATCT-3'; PDK1 forward, 5'CTGTGATACGGATCAGAAACCG-3', reverse, 5' -TCC ACCAAACAATAAAGAGTGCT-3'; PGK1 forward, 5'GAACAAGGTTAAAGCCGAGCC-3', reverse, 5' -GTG GCAGATTG-ACTCCTACCA-3'; MET forward, 5' -GGT TCACTGCATAT-TCTCCCC-3', reverse, 5'-ACCA TCTT-TCGTTTCCTTTAGCC-3'; GAPDH forward, 5'GCATTGCCCTCAACG-ACCAC-3', reverse: 5'-CCA C-CACCCTGTTGCTGTAG- $3^{\prime}$ and $\beta$-actin, forward $5^{\prime}$ ACTCGTCATACTCCTGCT-3', reverse 5'-GAAACT ACCTTCAACTCC-3'

\section{Western blotting (WB)}

The WB was conducted as reported previously ${ }^{14}$. Antibodies used were mouse anti-GAPDH (1:10,000, Abcam, ab8245), rabbit anti-NFAT5 (1:1000, Abcam, ab226308), and rabbit anti-PGK1 (1:500, Abcam, ab38007).

\section{Immunohistochemistry (IHC)}

IHC was performed as previously described ${ }^{14}$. AntiNFAT5 (1:100, Abcam, ab226308), PGK1 (1:100, Abcam, ab38007), and anti-PCNA (1:500, Cell Signaling Technology, \#13110) antibodies were used. Tumor tissues were scored according to the percentage of stained area $(0=$ $0 \%, \quad 1=1-10 \%, 2=11-50 \%, 3=51-80 \%$, and $4=$ $81-100 \%)$ and darkness of the nuclei or cytoplasm staining $(0=$ no staining, $1=$ weak staining, $2=$ moderate staining, and $3=$ strong staining). Final scores were determined by multiplying the 2 numbers mentioned above ("negative" for a score of 0 , "weak" for a score of 1-4, "moderate" for a score of 5-8, and "positive" for a score of 9-12). The high-expression group had scores greater than 5 , while the low-expression group scores were $\leq 5$.

\section{CCK-8 and colony formation assays}

Cell viability was measured using a Cell Counting Kit-8 (CCK-8, Dojindo Molecular Technologies, Japan). Cells were collected after stable transfection for $72 \mathrm{~h}$, and then they were seeded in plates (96-well) at a density of $2 \times 10^{4}$ cells $/ \mathrm{ml}$. CCK- 8 (10 $\mu \mathrm{l} /$ well $)$ reagent was added to each well at $0,1,2,3,4$, and 5 days. The absorbance at each wavelength of $450 \mathrm{~nm}$ was measured by an automatic enzyme-linked immune detector after $1 \mathrm{~h}$ of incubation. The cell growth curve was plotted with time on the horizontal axis and the OD value on the vertical axis. The experiment was repeated three times.

In the colony formation test, target cells were seeded into six-well plates. Two weeks later, the colonies were fixed with polyformaldehyde and stained with $0.5 \%(\mathrm{w} / \mathrm{v})$

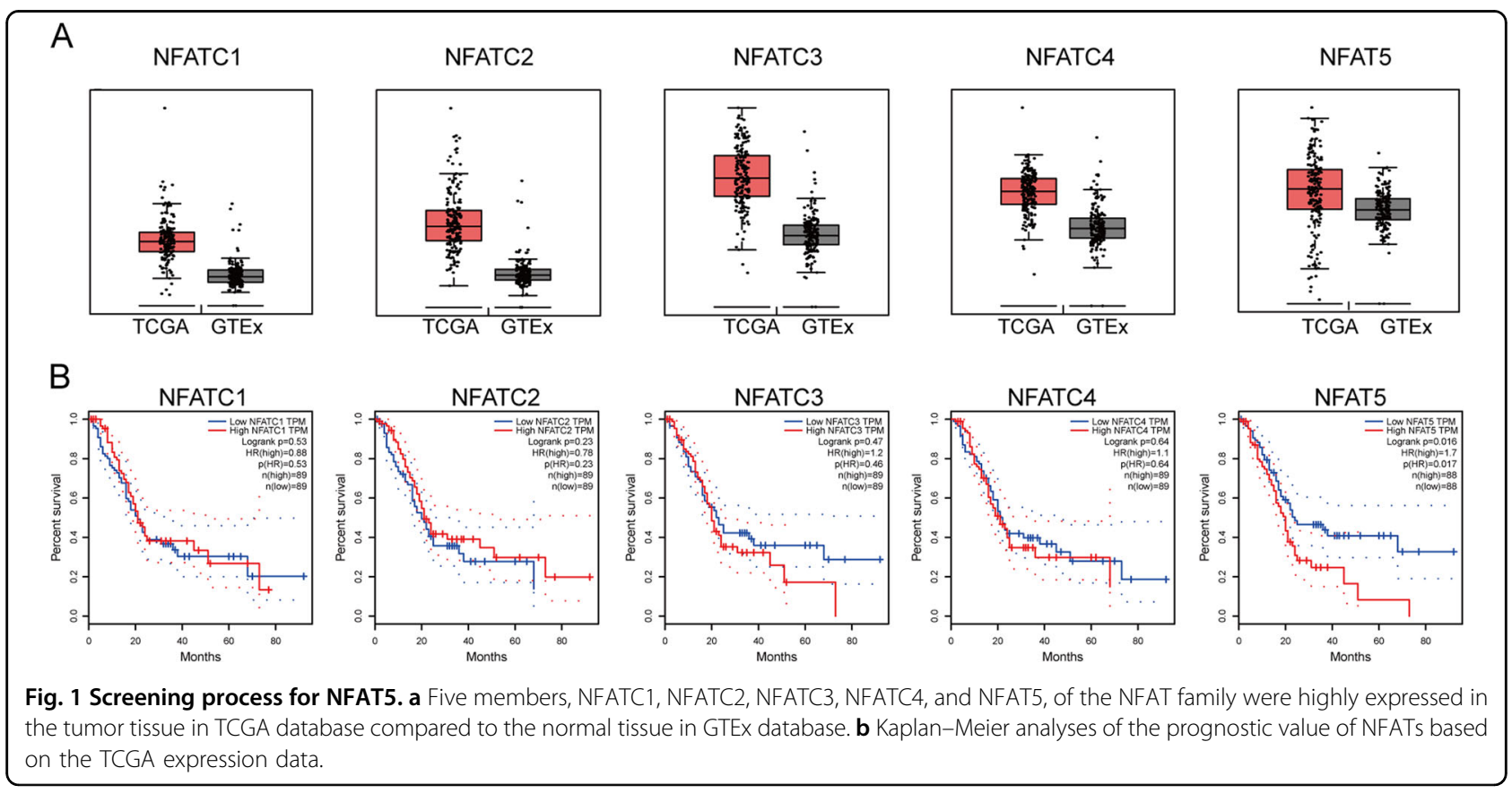


crystal violet. Photographs were taken and cell counts were calculated.

\section{Seahorse analyses}

The extracellular acidification rate (ECAR) and oxygen consumption rate (OCR) of the cells was assessed using the Seahorse XF96 Flux Analyser (Seahorse Bioscience,
Agilent). In brief, $1 \times 10^{4}$ cancer cells were seeded in an incubation plate as the protocol indicated. Cells were cultured at $37^{\circ} \mathrm{C}$ overnight for adhesion. Before detection, culture medium was replaced with assay media. The glycolytic stress test kit (Seahorse Cat. \#103020-100) and mitochondrial stress test kit (Seahorse Cat. \#103015-100) were purchased for ECAR and OCR

A GSE 15471

GSE 28735

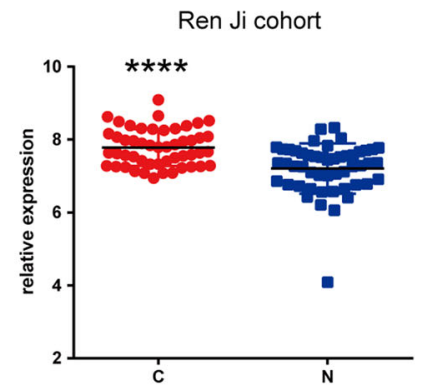

B
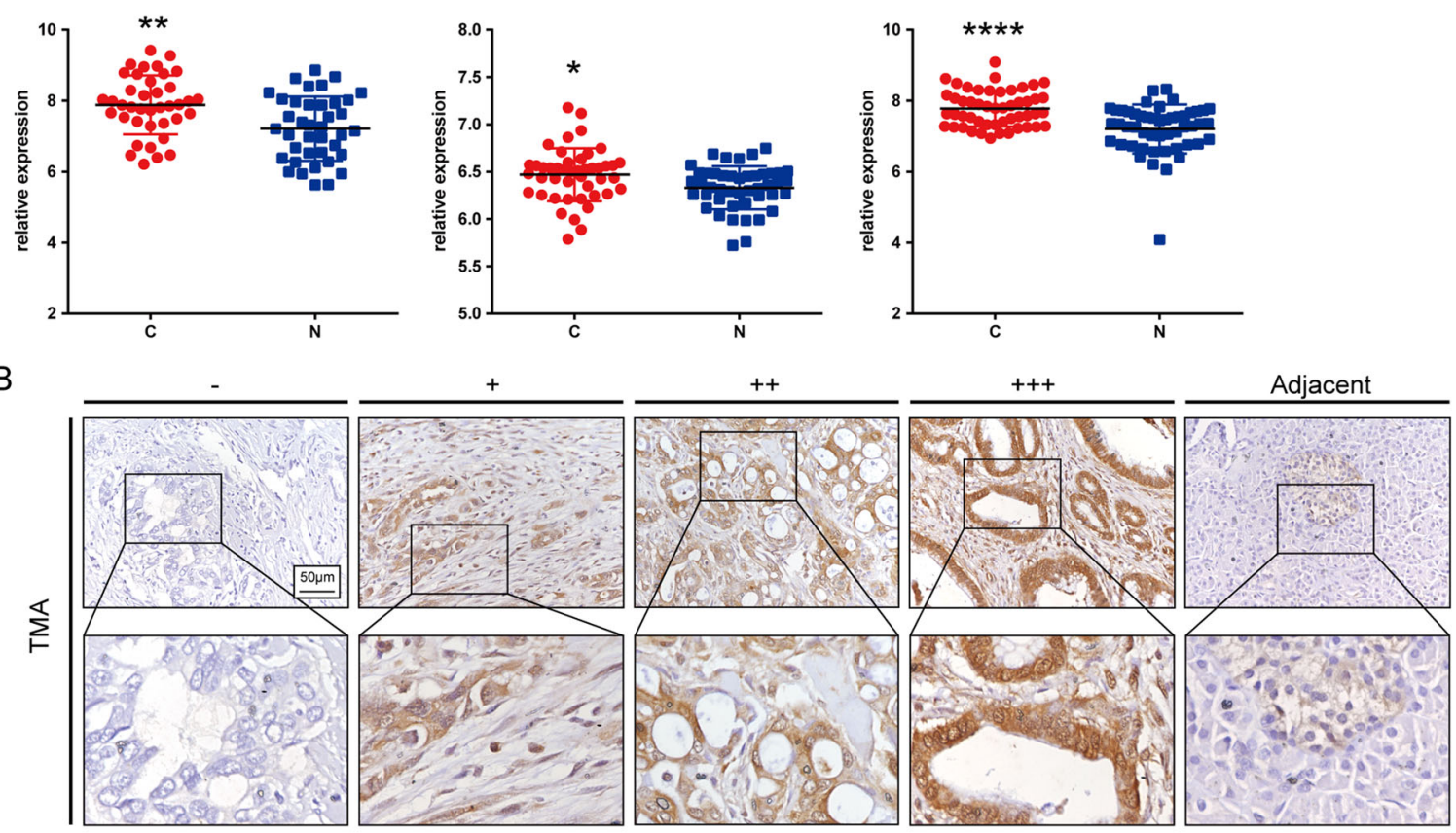

C

D
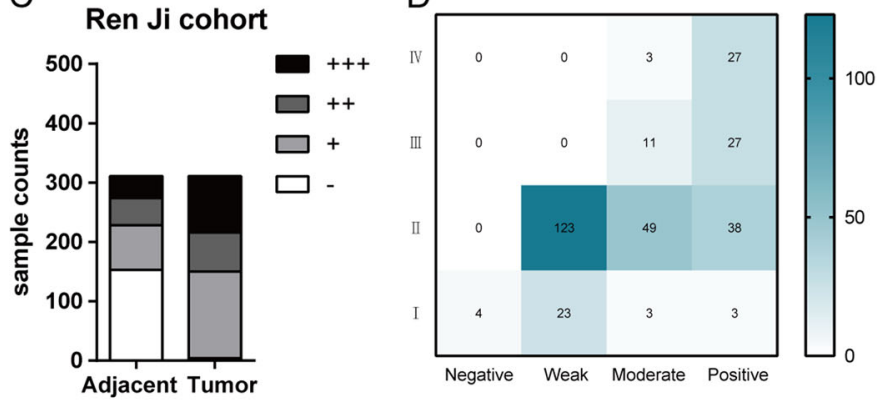

E

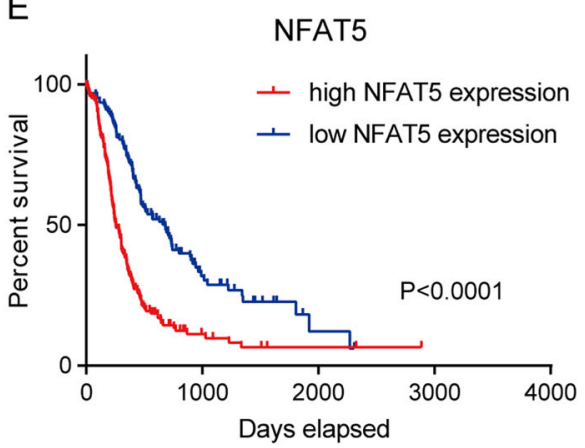

$\mathrm{F}$

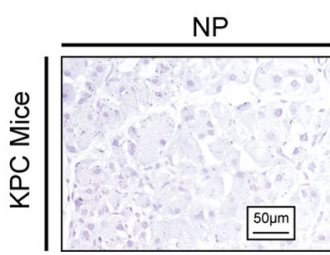

PanIN 1A-1B
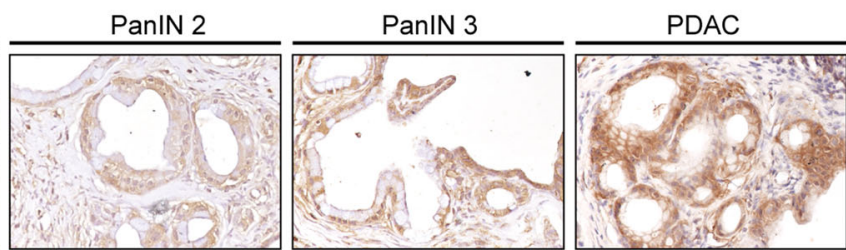

Fig. 2 High NFAT5 expression was linked to poor prognosis in PDAC. a The NFAT5 expression of tumor tissues and paired non-tumor tissues in the GSE15471, GSE28735, and Ren Ji cohort. b Standard immunohistochemical scoring pictures of NFAT5 expression in 311 pancreatic cancer tumors and adjacent normal tissues. $\mathbf{c}$ Respective sample counts in tumor tissues and adjacent tissues. $\mathbf{d}$ Heatmap shows the counted numbers of four levels of NFAT expression and its correlation with TNM classification according to the IHC. e Kaplan-Meier analysis of overall survival rate related to the expression of NFAT5 expression in 311 cases based on the Renji patients. $\mathbf{f}$ Standard immunohistochemical pictures in different stages of PDAC progression in KPC mice. ${ }^{*} P<0.05,{ }^{* *} P<0.01,{ }^{* * *} P<0.0001$. 
detection, respectively. The assays were performed using the manufacturer protocols.

\section{Glucose and lactate measurement}

To perform the glucose consumption rate and lactate production assays, cells were cultured in a six-well plate for $24 \mathrm{~h}$. The supernatants were then collected for further detection. The Amplex ${ }^{\circledast}$ Red Glucose/Glucose Oxidase Assay Kit (Invitrogen, Cat. \# A22189) and Lactate Assay Kit (BioVision, Cat. \# ABIN411683) were purchased for each assay.

PDAC transgenic model and orthotopic xenograft model

The PDAC transgenic model and orthotopic xenograft model were utilized, as reported previously ${ }^{15}$.

\section{Promoter activity analysis by dual luciferase assay}

Promoters of human PGK1 were cloned into pGL4.10Basic vector. Cells were seeded in 96-well plates at a density of $1.2 \times 10^{5}$ cells per well. The cells were transfected with vectors, either expressing wild-type or mutant PGK1 promoter using Lipofectamine 3000 (Thermo Fisher Scientific). Next, the cells were assayed for both firefly and Renilla luciferase activities using a dual luciferase system (Promega, Madison, WI, USA), as described in the manufacturer's protocol. The experiments were performed in triplicate and repeated three times. The data are presented as the fold-change relative to the control group.

\section{Statistics}

Data are presented as the means \pm SD. Statistical analyses were performed using SPSS 22.0 for Windows (IBM). Cumulative survival time was calculated by the Kaplan-Meier method and analyzed by the log-rank test. Correlation of NFAT5 expression with categorical clinical variables in patients with PDAC was evaluated by $\chi 2$ test or Fisher's exact test. Univariate and multivariate Cox regression analyses were performed to identify the factors
Table 1 Correlation between NFAT5 expression and clinicopathologic factors.

\begin{tabular}{|c|c|c|c|}
\hline \multirow[t]{2}{*}{ Factors } & \multicolumn{2}{|c|}{ Expression of NFAT5 } & \multirow[t]{2}{*}{$P$ value } \\
\hline & Low $(n=150)$ & High $(n=161)$ & \\
\hline \multicolumn{4}{|l|}{ Age (y) } \\
\hline$<65$ & $72(48 \%)$ & $73(45.3 \%)$ & 0.639 \\
\hline$\geq 65$ & $78(52 \%)$ & $88(54.7)$ & \\
\hline \multicolumn{4}{|l|}{ Sex } \\
\hline Male & $86(57.3 \%)$ & $90(55.9 \%)$ & 0.799 \\
\hline Female & $64(42.7 \%)$ & $71(44.1 \%)$ & \\
\hline \multicolumn{4}{|l|}{ Tumor size } \\
\hline$<2 \mathrm{~cm}$ & $26(17.3 \%)$ & $11(6.8 \%)$ & $0.004(* * *)$ \\
\hline$\geq 2 \mathrm{~cm}$ & 124 (82.7\%) & 150 (93.2\%) & \\
\hline \multicolumn{4}{|c|}{ Tumor differentiation } \\
\hline Moderate/poor & $102(68.0 \%)$ & $92(57.1 \%)$ & $0.048(*)$ \\
\hline Well & $48(32.0 \%)$ & 69 (42.9\%) & \\
\hline \multicolumn{4}{|c|}{ Lymph node metastasis } \\
\hline Absent & $113(75.3 \%)$ & $82(50.9 \%)$ & $<0.0001(* * *)$ \\
\hline Present & $37(24.7 \%)$ & $79(49.1 \%)$ & \\
\hline \multicolumn{4}{|c|}{ Distant metastasis } \\
\hline Absent & $150(100.0 \%)$ & $131(81.4 \%)$ & $<0.0001(* * *)$ \\
\hline Present & $0(0.0 \%)$ & $30(18.6 \%)$ & \\
\hline \multicolumn{4}{|l|}{ TNM stage } \\
\hline$|/| \mid$ & $150(100.0 \%)$ & $93(57.8 \%)$ & $<0.0001(* * * *)$ \\
\hline III/IV & $0(0.0 \%)$ & $68(42.2 \%)$ & \\
\hline \multicolumn{4}{|l|}{ Nerve invasion } \\
\hline Absent & $88(58.7 \%)$ & $77(47.8 \%)$ & 0.056 \\
\hline Present & $62(41.3 \%)$ & $84(52.2 \%)$ & \\
\hline
\end{tabular}

${ }^{*} p<0.05,{ }^{* * *} p<0.01, * * * * p<0.0001$

Table 2 Univariate and Multivariate Cox regression analysis of potential prognostic factors in gastric cancer.

\begin{tabular}{|c|c|c|c|c|}
\hline \multirow[t]{2}{*}{ Factors } & \multicolumn{2}{|l|}{ Univariate } & \multicolumn{2}{|l|}{ Multivariate } \\
\hline & $\mathrm{HR}(95 \% \mathrm{Cl})$ & $P$ value & $\mathrm{HR}(95 \% \mathrm{Cl})$ & $P$ value \\
\hline Age & $1.314(1.000-1.727)$ & 0.05 & $1.467(1.103-1.951)$ & 0.008 \\
\hline Gender & $0.861(0.655-1.132)$ & 0.861 & & \\
\hline Tumor size & $1.682(1.070-2.644)$ & 0.024 & $1.240(0.780-1.969)$ & 0.363 \\
\hline Tumor differentiation & $1.682(1.274-2.222)$ & $<0.0001\left(^{* * * *}\right)$ & $1.392(1.043-1.857)$ & $0.025\left(^{*}\right)$ \\
\hline Lymph node metastasis & $1.686(1.279-2.223)$ & $<0.0001\left(^{* * * *}\right)$ & $1.504(1.120-2.021)$ & $0.007(* * *)$ \\
\hline Distant metastasis & $1.858(1.201-2.874)$ & $<0.005(* * *)$ & $1.227(0.688-2.185)$ & 0.488 \\
\hline TNM stage & $1.827(1.326-2.518)$ & $<0.0001\left(^{* * * *}\right)$ & $1.003(0.645-1.560)$ & 0.991 \\
\hline Nerve invasion & $1.147(0.876-1.502)$ & 0.319 & & \\
\hline NFAT5 experssion & $2.410(1.821-3.190)$ & $\left.<0.00011^{(* * * *}\right)$ & $2.031(1.474-2.798)$ & $<0.0001\left(^{* * * *}\right)$ \\
\hline
\end{tabular}

${ }^{*} p<0.05,{ }^{* * *} p<0.01,{ }^{* * * *} p<0.0001$ 


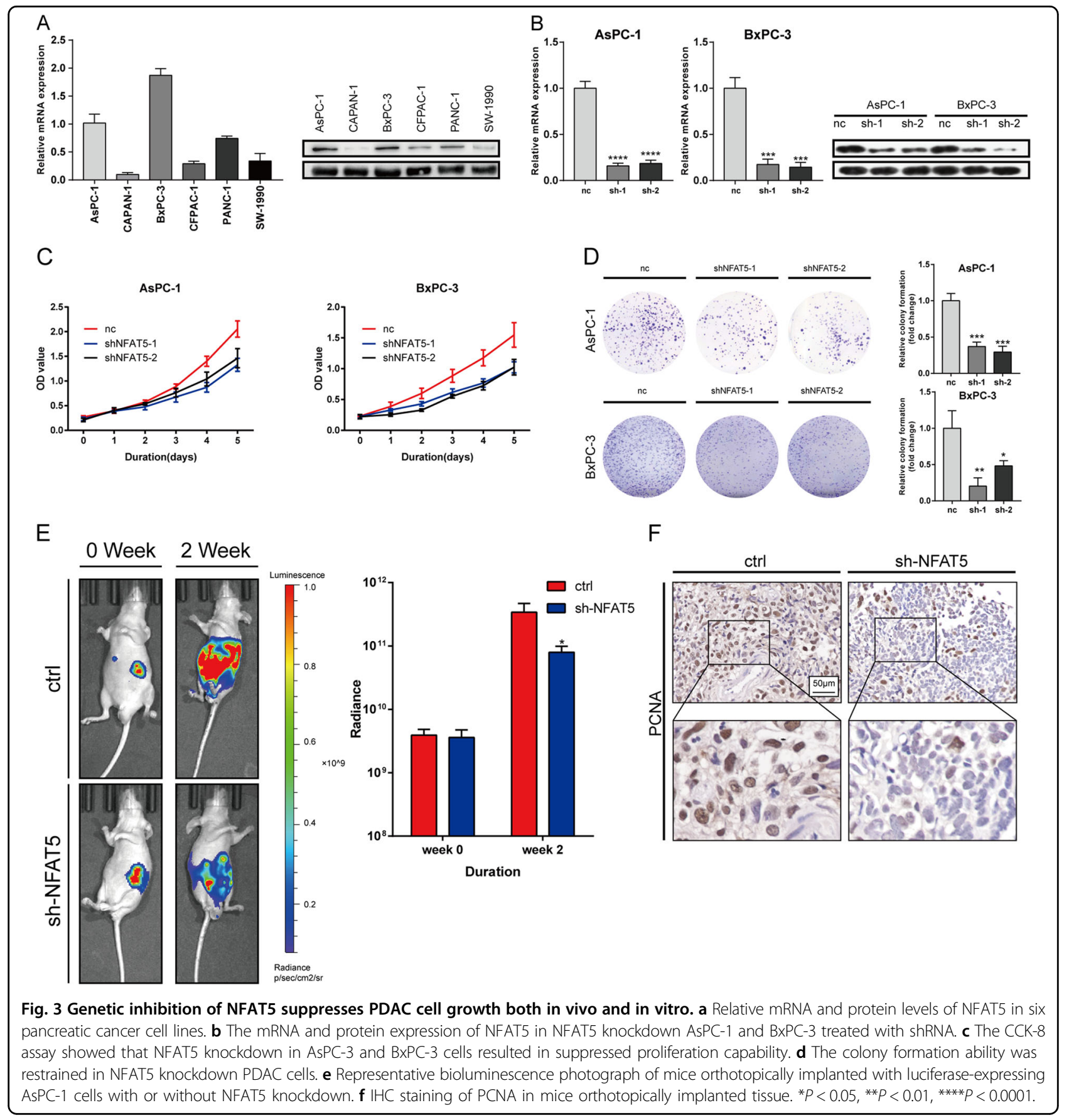

that had a significant influence on survival by Cox proportional hazards model. The student's $t$-test or one-way ANOVA was used for comparison between groups. Values of $P<0.05$ were considered statistically significant.

\section{Results}

Oncogene screening of NFAT transcription factor family in PDAC

To screen for the potential oncogene in the NFAT family, The Cancer Genome Atlas (TCGA) and The
Genotype-Tissue Expression (GTEx) datasets were collected and analyzed. As shown in Fig. 1a, NFATC1, NFATC2, NFATC3, NFATC4, and NFAT5 were all highly expressed in the tumor tissue of the TCGA dataset compared with normal tissue of the GTEx dataset. However, in the TCGA dataset, only increased NFAT5 predicted poor prognosis (Fig. 1b), so we further investigated its role in PDAC. We first examined the NFAT5 expression in three Gene Expression Omnibus (GEO) datasets including our own Renji cohort. The results 


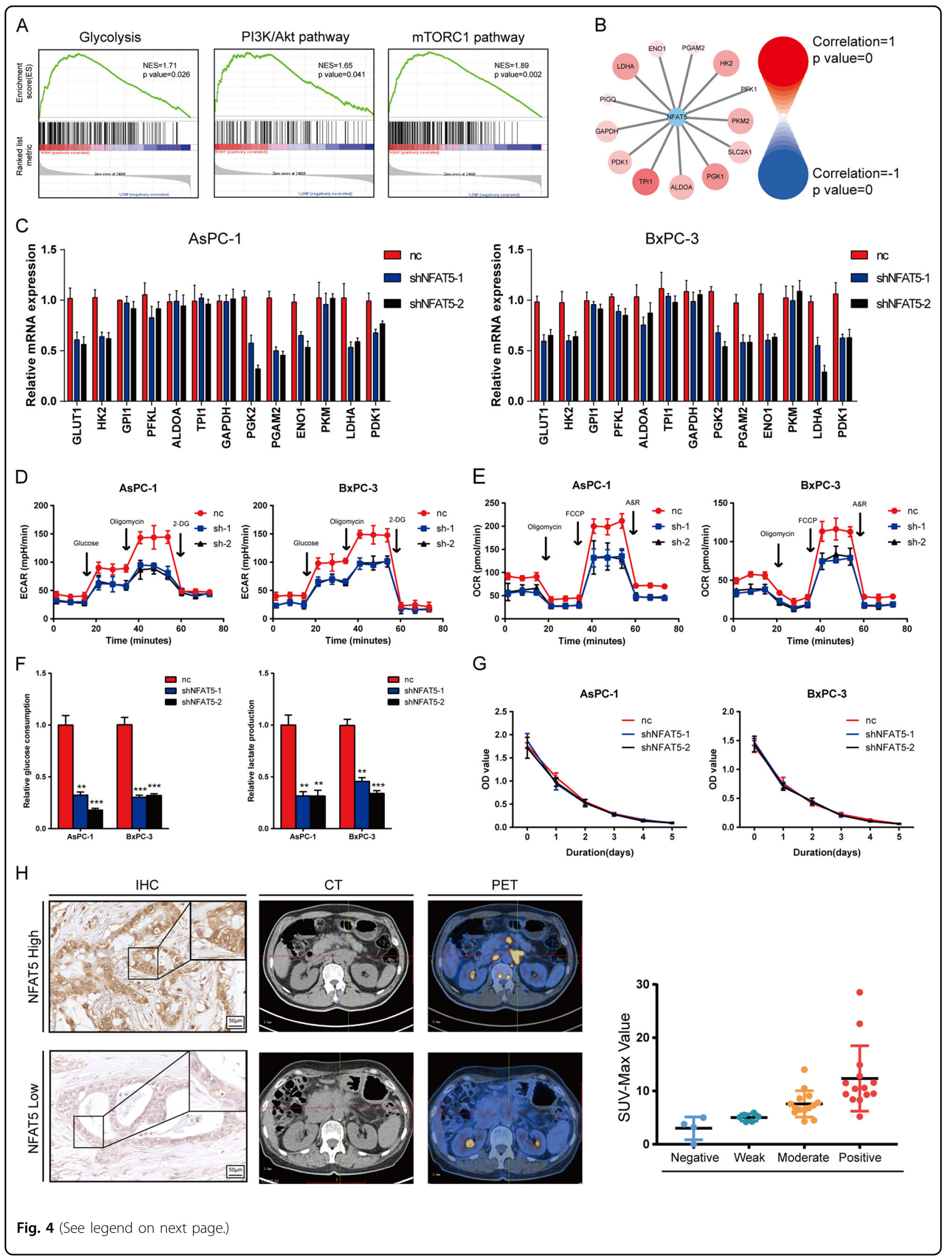


(see figure on previous page)

Fig. 4 NFAT5 promoted PDAC cell growth by enhancing the Warburg effect. a Gene set enrichment analysis (GSEA) of the NFAT5 highexpression group and the low-expression group using hallmark gene sets showed the relationship between NFAT5 and three datasets closely related to the Warburg effect. NES, normalized enrichment score. $\mathbf{b}$ The correlation between NFAT5 and glycolytic enzymes. c Relative mRNA levels of glycolysis-related genes of PDAC with or without NFAT5 knockdown. d, e Glycolytic function and mitochondrial stress test of NFAT5 knockdown AsPC-1 and BxPC-3 cells treated with shRNA were measured by extracellular acidification rate (d) and oxygen consumption rate (e), respectively. f Relative glucose consumption and lactate production in normal and NFAT5 knockdown AsPC-1 and BxPC-3 cell lines. g NFAT5 knockdown AsPC-1 and $\mathrm{BxPC}-3$ cell proliferation capability indicated no difference after glucose was replaced with galactose compared to normal AsPC-1 and BxPC-3 cells. $\mathbf{h}$ Typical immunohistochemical images of NFAT5 low and high-expression group along with their CT and PET-CT images. The relationship between 4 NFAT5 expression groups and SUV-Max Values was counted. Assays shown in Fig. 4c, f were performed in hypoxia condition. ${ }^{*} P<0.05$, ${ }^{* *} P<0.01,{ }^{* * * *} P<0.0001$.

showed that NFAT5 manifested a higher expressed pattern compared with adjacent noncancerous tissue in all three datasets (Fig. 2a). We then validated our results on the protein level by performing IHC staining in a Renji TMA containing 311 pathologist-verified PDAC specimens with paired corresponding adjacent pancreatic tissue. The expressions were scored based on staining intensity and area (Fig. 2b). As shown in Fig. 2c-e, tumor tissue manifested a higher staining score, and patients with increased NFAT5 had a poorer prognosis. We then performed Chi-square analysis to evaluate the relationship between the expression of NFAT5 and clinicopathologic features of samples in the TMA cohort. The result showed that NFAT5 was significantly correlated with tumor size, tumor differentiation, lymph node metastasis, distant metastasis, and TNM stage (Table 1). We also used univariate and multivariate analysis to identify NFAT5 expression as an independent risk factor for PDAC (Table 2). We also detected the expression pattern of NFAT5 in KrasG12D/+/Trp-53R172H/+/Pdx1-Cre (KPC) mice. As shown in Fig. 2f, the staining of NFAT5 is positively correlated with the progression of PDAC. These data suggest that NFAT5 may play a role as an oncogene in PDAC.

\section{Genetic inhibition of NFAT5 suppresses PDAC cell growth both in vivo and in vitro}

To study the effect of NFAT5 on PDAC, we examined the expression level of NFAT5 in six pancreatic cancer cell lines both at the mRNA and protein level, at which AsPC1 and BxPC-3 exhibit the highest expression (Fig. 3a). Two cell lines were selected for knockdown assay, which was assessed at the mRNA and protein level (Fig. 3b). We then performed CCK8 assays and colony formation assays to evaluate the pro-proliferation effect of NFAT5 on tumor cells (Fig. 3c, d). The orthotopic xenograft model was established to test the effect of NFAT5 in vivo. As shown in Fig. 3e, mice injected with NFAT5 knockdown cell lines (AsPC-1) occupied lower bioluminescent emission compared with the control group (AsPC-1). The IHC result for specimens also indicated that the proliferation of the control group was stronger than that of the NFAT5 knockdown group (Fig. 3f).

\section{NFAT5 facilitates PDAC cell survival via contributing to the Warburg effect}

To understand how NFAT5 influences pancreatic tumor cell growth, we first performed Geneset Enrichment Analysis (GSEA) by dividing samples into two groups based on NFAT5 expression in the Renji cohort. As shown in Fig. 4a, three datasets closely related to the Warburg effect (glycolysis, PI3K/AKT/mTOR signaling, and mTORC1 signaling) were enriched in the NFAT5 high-expression group, indicating that NFAT5 may have an impact on glucose metabolism in pancreatic cancer. We then assessed the correlation between NFAT5 and several key enzymes in the Warburg effect in datasets. As shown in Fig. 4b, almost all enzymes are positively correlated with NFAT5, suggesting that NFAT5 upregulates the Warburg effect in PDAC. To further validate the underlying reprogramed glucose metabolism modulated by NFAT5, we performed qPCR to investigate the effect of NFAT5 on glycolysis-related genes and found that knockdown of NFAT5 results in impaired expression of HK2, PGK1, and LDHA (Fig. 4c). Cells were cultured in hypoxia condition to simulate the hypovascular tumor microenvironment in PDAC. The extracellular acid rate (ECAR) and OCR of PDAC cells were measured by the Seahorse XF96 Flux Analyzer. The result showed that knockdown of NFAT5 significantly decreased both ECAR and OCR in two cell lines (Fig. 4d, e), indicating that NFAT5 plays a contributing role in glucose metabolism in PDAC. In addition, knockdown of NFAT5 also led to a marked decrease in glucose uptake and extracellular lactate levels in both AsPC-1 and BxPC-3 cell lines (Fig. 4f). We then aimed to investigate whether the effect of NFAT5 on PDAC glucose metabolism could facilitate the cell survival. To test our hypothesis, we replaced the glucose in culture medium with galactose, which blocks the glucose flux to eliminate the Warburg effect on cellular function. Just as anticipated, reduced glucose flux greatly compromised the pro-survival effect of NFAT5 (Fig. 4g). Then, we tested the expression status of NFAT5 by IHC staining and analyzed its correlation with $18 \mathrm{~F}$ FDG uptake in 39 pancreatic cancer patients who received PET-CT examination to validate our results in clinical 
A

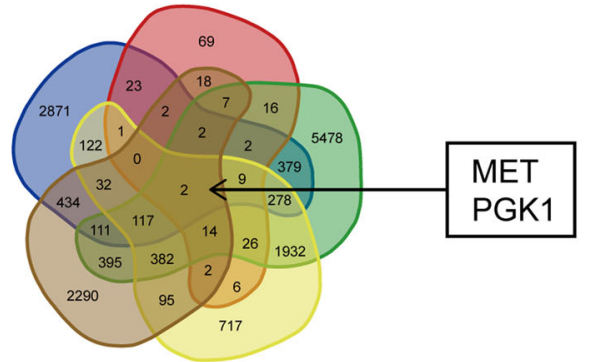

Potential target genes prediceted by GTRD

Differential expressed genes in Ren Ji cohort

Glycolysis marker genes

Glycolysis-related genes in Ren Ji cohort

Significant in prognosis prediction.
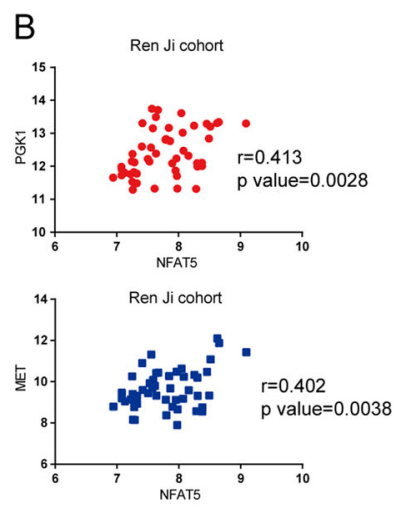
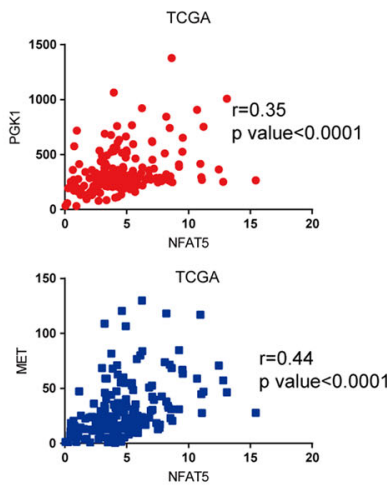

C

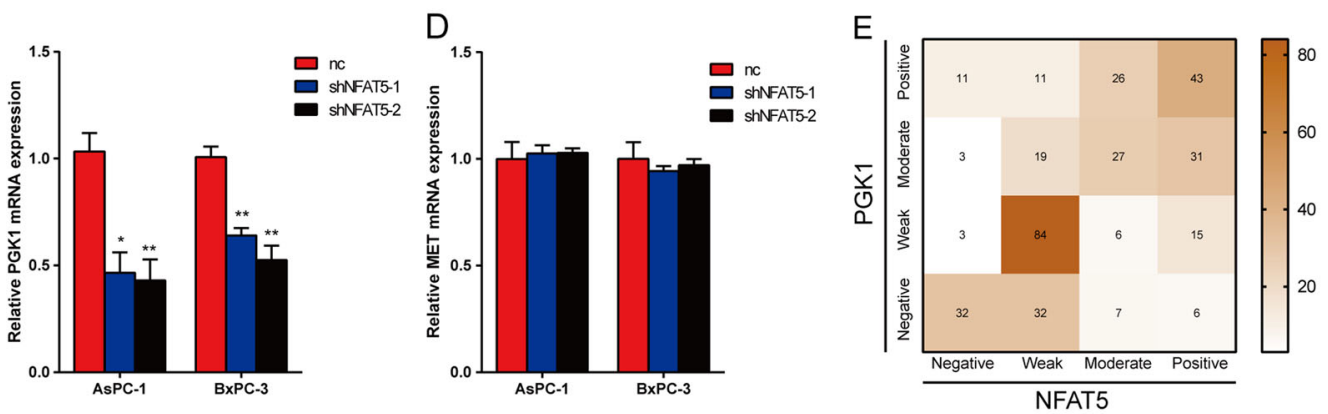

$\mathrm{F}$

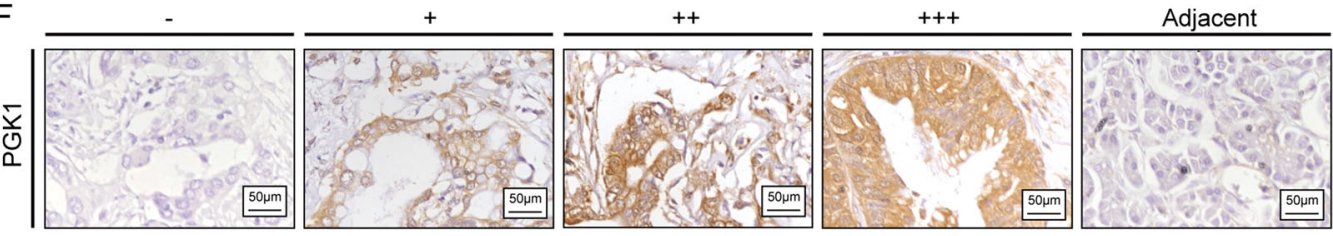

G
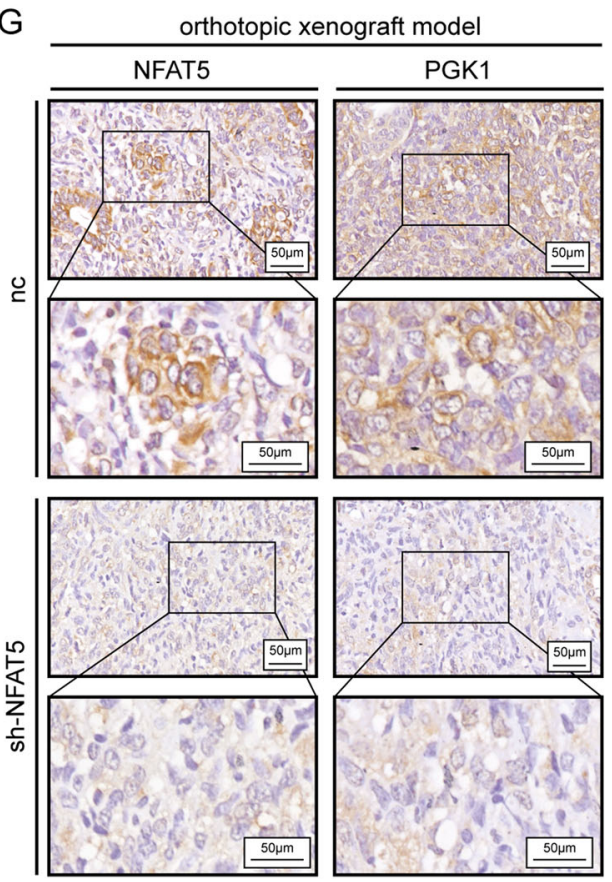

$\mathrm{H}$
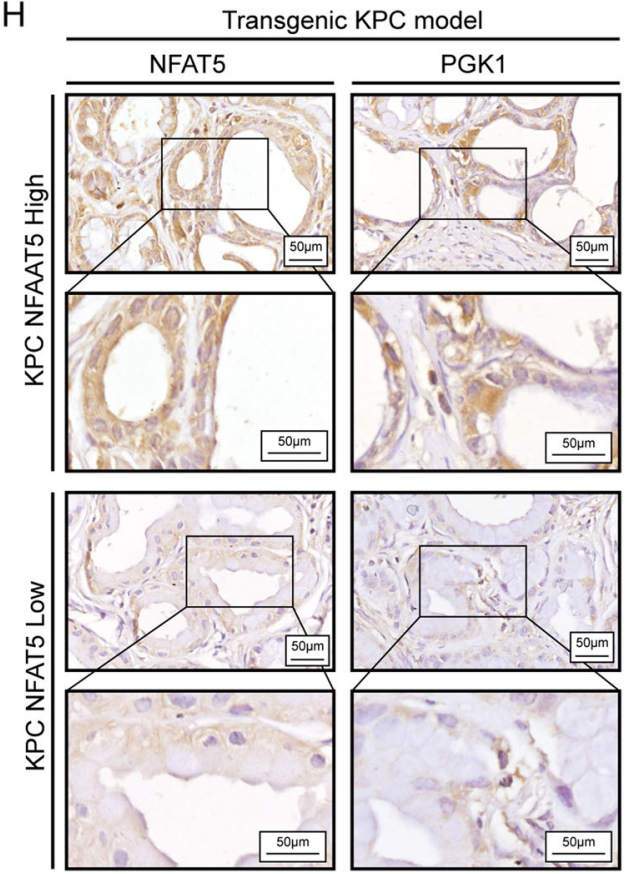

Fig. 5 (See legend on next page.) 
(see figure on previous page)

Fig. 5 PGK1 is a direct target gene of NFAT5. a Venn diagram showing the intersection of five gene sets. $\mathbf{b}$ The relevance of NFAT5 with PGK1 and MET is displayed in the Renji cohort and TCGA database. $\mathbf{c}$, d Relative PGK1 and MET mRNA expression in the control and NFAT5 knockdown PDAC cell lines. e Heatmap showing the counted numbers of four levels of PGK1 expression and its correlation with NFAT5. $\mathbf{f}$ Standard immunohistochemical scoring pictures of PGK1 expression in 311 pancreatic cancer tumors and adjacent normal tissues. $\mathbf{g}$ Respective IHC staining of NFAT5 and PGK1 in orthotopic xenograft models. $\mathbf{h}$ Respective IHC staining of NFAT5 and PGK1 in KPC mice. Assays shown in Fig. $5 c$, $d$ were performed in hypoxia condition. ${ }^{*} P<0.05,{ }^{* *} P<0.01,{ }^{* * *} P<0.0001$.

A

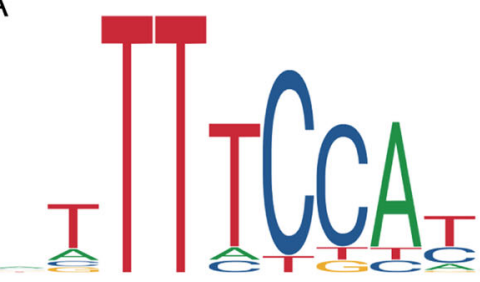

Canonical NFAT5 bingding site

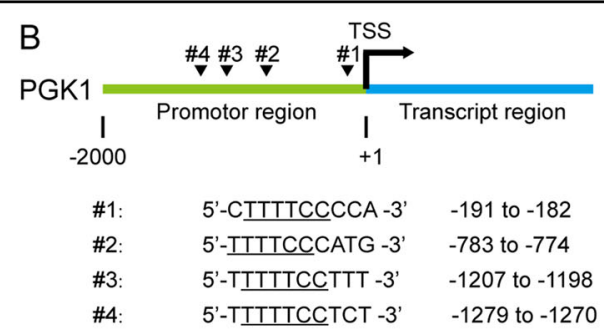

C

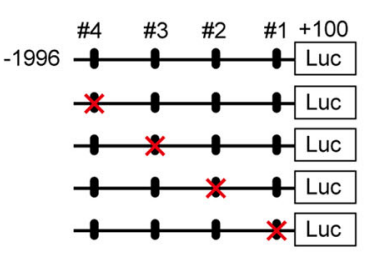

AsPC-1

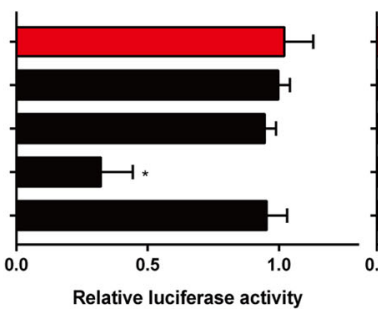

BxPC-3

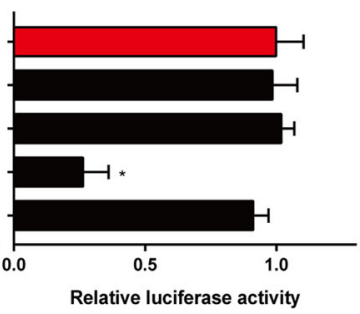

\#4. 5'-TTTTCC -3' Mutation: 5 - - GCGCAT-3

\#3: 5 '-TTTTCC $-3^{\prime}$ Mutation: 5 '-GCGCAT-3'

\#2: 5 '-TTTTCC -3 '

Mutation: 5 '- $\overline{\text { GCGCAT-3 }}$

\#1: 5 - TTTTCC $-3^{\prime}$ Mutation: 5 - -GCGCAT-3

Fig. 6 PGK1 is a direct target gene of NFAT5. a The sequence logo graph manifested the canonical binding site of NFAT5 predicted by JASPAR. b Four potential binding sequences were found in the PGK1 promoter region. c Mutated binding sites of NFAT5 lead to impaired luciferase activity in two cell lines. ${ }^{*} P<0.05$, ${ }^{* *} P<0.01,{ }^{* * * *} P<0.0001$.

samples. The results demonstrated that higher NFAT5 expression was accompanied with a higher standard uptake value (SUV-Max) (Fig. 4h). These data demonstrate that NFAT5 facilitates PDAC cell survival via contributing to the Warburg effect.

\section{PGK1 is a direct target gene of NFAT5}

To further understand the underlying mechanism of how NFAT5 contributes to the Warburg effect, as shown in Fig. 5a, several conditions (1) Predicated by the Gene Transcription Regulation Database (GTRD), (2) Differentially expressed in the Renji cohort, (3) Glycolysis marker genes, (4) Top 10\% glycolysis-related genes in the Renji cohort, and (5) Significant in prognosis predication) were used to screen for the downstream target genes of NFAT5. MET and PGK1 were then used for subsequent research. We first calculated the correlation between NFAT5 and two target genes in both the Renji cohort and TCGA, which showed that NFAT5 is strongly positively correlated with MET and PGK1 in two datasets (Fig. 5b). We then performed qRT-PCR to further investigate whether knockdown of NFAT5 would decrease the expression of MET or PGK1. As shown in Fig. 5c, d, the expression of PGK1 was significantly decreased in NFAT5 knockdown cell lines, while MET did not exhibit evident differences in different cell lines. To further validate the correlation between PGK1 and NFAT5, we detected the expression of PGK1 in the TMA and found that it was positively correlated with the expression of NFAT5 (Fig. 5e, f. This result was also validated in transgenic models and orthotopic xenograft models (Fig. 5g, h). We then preformed a luciferase assay to investigate whether NFAT5 directly influenced the expression of PGK1. We searched for the canonical binding site of NFAT5 predicted by JASPAR in the promoter region of the PGK1 coding sequence, and four potential binding sites emerged (Fig. 6a, b). After respectively mutating all these four potential binding sites, the luciferase activity of the cells transduced with the third binding site mutant plasmids was significantly lower than that of the other groups in both cell lines (Fig. 6c). Collectively, these data suggest that PGK1 is the direct target gene of NFAT5 in PDAC. 


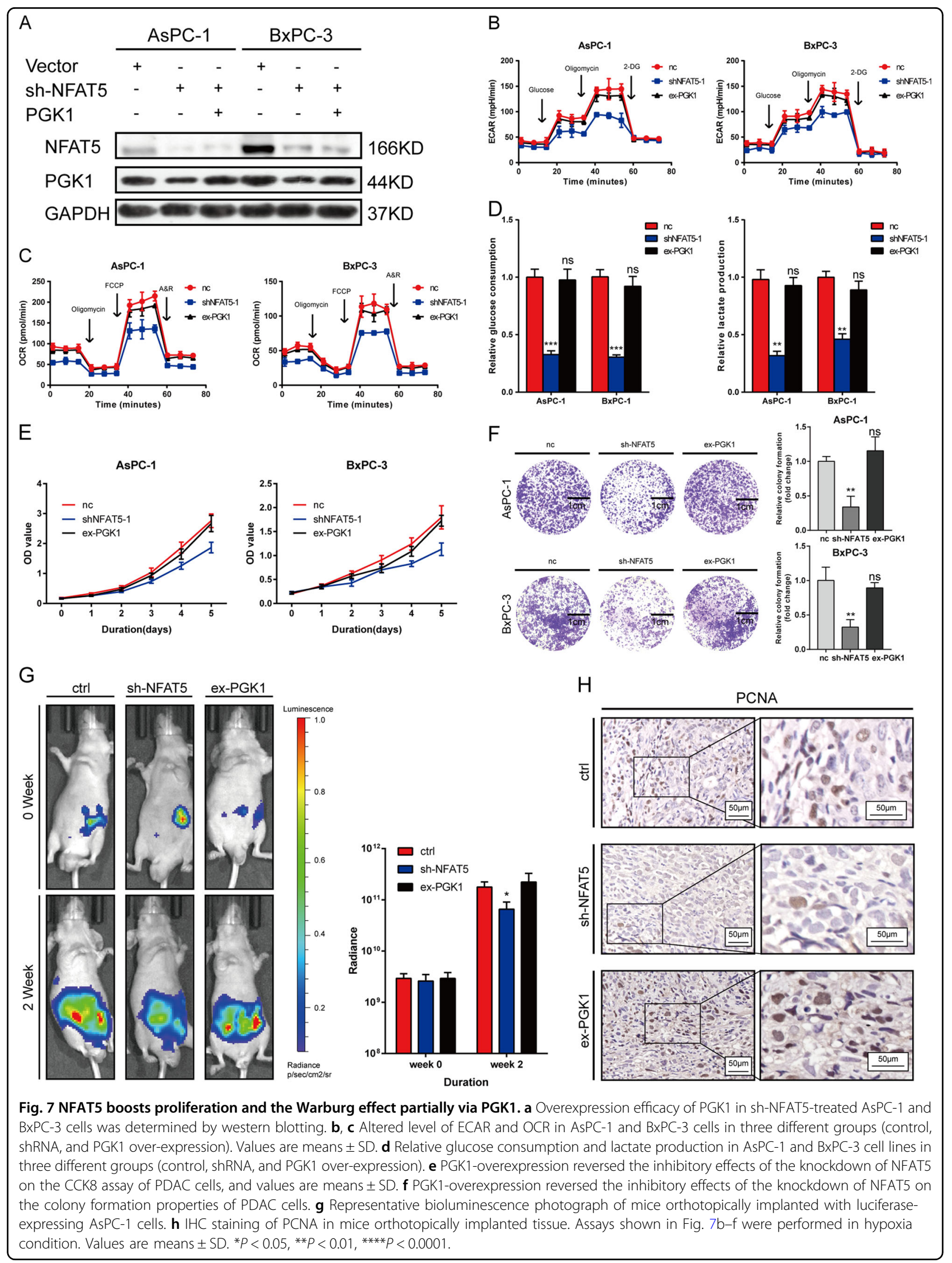




\section{NFAT5 suppresses proliferation and the Warburg effect by inhibiting PGK1 expression}

To investigate whether NFAT5 performs a pro-cancer function via PGK1, we over-expressed PGK1 in AsPC-1 and BxPC-3 with knockdown of NFAT5. The efficiency was confirmed by WB (Fig. 7a). As revealed by Seahorse ECAR and OCR measurements, expression of PGK1 rescued the Warburg effect in PDAC cells (Fig. 7b, c). Glucose consumption and lactate production assays were performed to validate these results (Fig. 7d). In addition, the pro-survival function of NFAT5 was also compromised with over-expression of PGK1, demonstrated by CCK8 and colony formation assays (Fig. 7e, f). The orthotopic xenograft model was established to test the effect of NFAT5 in vivo. As shown in Fig. $7 \mathrm{~g}$, overexpression of PGK1 rescued the decreased bioluminescent emission caused by NFAT5 knockdown. IHC staining of the decreased bioluminescent emission caused by NFAT5 knockdown. IHC staining of PCNA indicated the same result (Fig. 7h).

\section{Discussion}

Since its discovery in the 1920s, the Warburg effect has been widely proven to contribute to malignancy progression and defined as one of the hallmarks of cancer ${ }^{16}$. Via utilizing the Warburg effect, tumor cells generate energy promptly, and the intermediate products of glycolysis are used as proliferation fuel. PDAC has a wellacknowledged mesenchyme-abundant tumor microenvironment (TME), in which the Warburg effect is more crucial for cell survival and proliferation than in a mesenchyme-infertile TME. Therefore, targeting the Warburg effect could be a potential method for PDAC treatment and enhancing other anticancer therapies ${ }^{14,15,17}$.

Here, we demonstrated that transcription factor NFAT5 acts as an oncogene in PDAC through enhancing glycolysis of PDAC cells. The transcription factors of NFAT include NFATC1, NFATC2, NFATC3, NFATC4, and NFAT5, of which only NFAT5 is a significant prognostic factor. Zhou et al. demonstrated that NFAT5 downregulates $\mathrm{mTORC} 1$ signaling in intestinal cells ${ }^{18}$, while we found that NFAT5 is positively correlated with mTOR pathway in PDAC both in vivo and in vitro which is consistent with Hassas's work ${ }^{19}$. The role of NFAT5 has be extensively studied in the immune system, with little work related to cancer that has been reported ${ }^{20-22}$. In our study, we found that NFAT5 functions as an oncogene in PDAC. Firstly, we showed that the expression of NFAT5 is higher in several datasets both at the protein and mRNA level. Then, we observed that knockdown of NFAT5 leads to a decrease in ECAR and OCR, which further leads to impaired proliferation and colony formation. PET-CT is widely used in clinical diagnosis, in which the 18F-FDG uptake rate is measured and visualized as SUV-MAX. It can reflect the glucose metabolism level of the patient's tissue. By co-analyzing with IHC results of the same sample, we can assess and validate the influence of different genes on the Warburg effect in clinical PDAC patients. In this study, we showed that with high expression of NFAT5, patients are more likely to have a high SUV-MAX value, which validated our hypothesis that NFAT5 does contribute to the glycolysis of PDAC.

We then set several conditions to find the potential target genes of NFAT5 that affect the glycolysis of PDAC cells. PGK1, a key enzyme in the glycolysis pathway, emerged. PGK1 is the first enzyme generating ATP in glycolysis and is proven to be of vital importance in the progression of several types of cancer ${ }^{23-25}$. In our study, the regulation of PGK1 by NFAT5 was predicted by qRT$\mathrm{PCR}$, and the binding site was predicted in the promoter region, from which more than four binding sites were found and validated by the luciferase assay. Overexpression of PGK1 was then performed for the rescue assays. We found that the metabolic and proliferation deficiency caused by NFAT5 knockdown were compromised to a certain degree, indicating that PGK1 is one of the major target genes of NFAT5 that leads to PDAC progression.

In conclusion, our work first demonstrated that the expression of NFAT5 is negatively correlated with PDAC patient prognosis. Aberrant high expression of NFAT5 inhibits tumor proliferation and progression by dampening its Warburg effect, which is partly caused by transactivation of PGK1. Targeting the metabolic process of cancer cells may be a hopeful way in tumor treatment ${ }^{26}$. Our work presents novel insight into the pathology of PDAC and a potential target for new therapeutic strategies.

\section{Acknowledgements \\ This work was supported by grants from National Natural Science Foundation of China Project 81401931, Project 81502489, Project 81602414, Project 81802317, Project 81672358, Cultivating Funds of Renji Hospital, School of Medicine, Shanghai Jiao Tong University, China Project RJZZ18-012, Shanghai Municipal Education Commission-Gaofeng Clinical Medicine Grant Support (20181708, to Z.-G.Z.), Program of Shanghai Academic/Technology Research Leader (19XD1403400, to Z.-G.Z.), Science and Technology Commission of Shanghai Municipality (18410721000, to Z.-G.Z.) and Shanghai Municipal Health Bureau (2018BR32, to Z.-G.Z.).}

\section{Author details}

'Department of Biliary-Pancreatic Surgery, Ren Ji Hospital, School of Medicine, Shanghai Jiao Tong University, Shanghai, P. R. China. ${ }^{2}$ Department of General Surgery, Rui Jin Hospital, School of Medicine, Shanghai Jiao Tong University, Shanghai, P. R. China. ${ }^{3}$ Qingdao Women and Children's Hospital, Qingdao, China. ${ }^{4}$ Department of Hepatobiliary Pancreas Surgery, Shanghai East Hospital, Tong Ji University School of Medicine, Shanghai, P. R. China. ${ }^{5}$ State Key Laboratory of Oncogenes and Related Genes, Shanghai Cancer Institute, Ren Ji Hospital, School of Medicine, Shanghai Jiao Tong University, Shanghai, P. R. China 


\section{Conflict of interest}

The authors declare that they have no conflict of interest.

\section{Publisher's note}

Springer Nature remains neutral with regard to jurisdictional claims in published maps and institutional affiliations.

Supplementary information accompanies this paper at https://doi.org/ 10.1038/s41419-019-2072-5.

Received: 3 April 2019 Revised: 12 September 2019 Accepted: 2 October 2019

Published online: 11 December 2019

\section{References}

1. Costello, E., Greenhalf, W. \& Neoptolemos, J. P. New biomarkers and targets in pancreatic cancer and their application to treatment. Nat. Rev. Gastroenterol. Hepatol. 9, 435-444 (2012).

2. Siegel, R. L., Miller, K. D. \& Jemal, A. Cancer statistics, 2019. CA Cancer J. Clin. 69 7-34 (2019)

3. Guo, X. \& Cui, Z. Current diagnosis and treatment of pancreatic cancer in China. Pancreas 31, 13-22 (2005)

4. Long, J. et al. Cancer statistics: current diagnosis and treatment of pancreatic cancer in Shanghai, China. Cancer Lett. 346, 273-277 (2014).

5. Shaw, J. P. et al. Identification of a putative regulator of early $T$ cell activation genes. Science. 1988. 241: 202-205. J. Immunol. 185, 4972-4975 (2010).

6. Lopez-Rodriguez, C. et al. Loss of NFAT5 results in renal atrophy and lack of tonicity-responsive gene expression. Proc. Natl Acad. Sci. USA 101, 2392-2397 (2004).

7. Lee, J. H. et al. NFAT5 induction and its role in hyperosmolar stressed human limbal epithelial cells. Invest. Ophthalmol. Vis. Sci. 49, 1827-1835 (2008).

8. Kim, D. H., Kim, K. S. \& Ramakrishna, S. NFAT5 promotes in vivo development of murine melanoma metastasis. Biochem. Biophys. Res. Commun. 505 748-754 (2018)

9. Qin, X. et al. Upregulation of DARS2 by HBV promotes hepatocarcinogenesis through the miR-30e-5p/MAPK/NFAT5 pathway. J. Exp. Clin. Cancer Res. 36 148 (2017).

10. Qin, X. et al. NFAT5 inhibits invasion and promotes apoptosis in hepatocellular carcinoma associated with osmolality. Neoplasma 64, 502-510 (2017).
11. Alfarouk, K. O. et al. Glycolysis, tumor metabolism, cancer growth and dissemination. a new pH-based etiopathogenic perspective and therapeutic approach to an old cancer question. Oncoscience 1, 777-802 (2014).

12. Dougan, S. K. The pancreatic cancer microenvironment. Cancer J. 23, 321-325 (2017).

13. Liberti, M. V. \& Locasale, J. W. The Warburg Effect: how does it benefit cancer cells?. Trends Biochem. Sci. 41, 211-218 (2016).

14. Jiang, S. H. et al. Increased serotonin signaling contributes to the Warburg effect in pancreatic tumor cells under metabolic stress and promotes growth of pancreatic tumors in mice. Gastroenterology 153, 277-291.e19 (2017).

15. Hu, L. P. et al. Targeting purinergic receptor $\mathrm{P}_{2} \mathrm{Y} 2$ prevents the growth of pancreatic ductal adenocarcinoma by inhibiting cancer cell glycolysis. Clin. Cancer Res. 25, 1318-1330 (2019).

16. Hanahan, D. \& Weinberg, R. A. Hallmarks of cancer: the next generation. Cell 144, 646-674 (2011).

17. Zhang, Q. et al. Thymidine phosphorylase promotes malignant progression in hepatocellular carcinoma through pentose Warburg effect. Cell Death Dis. 10, 43 (2019).

18. Zhou, Y. et al. Nuclear factor of activated T-cells 5 increases intestinal goblet cell differentiation through an mTOR/Notch signaling pathway. Mol. Biol. Cell 25, 2882-2890 (2014)

19. Hassan, Z. et al. MTOR inhibitor-based combination therapies for pancreatic cancer. Br. J. Cancer 118, 366-377 (2018).

20. Remo, A. et al. Systems biology analysis reveals NFAT5 as a novel biomarker and master regulator of inflammatory breast cancer. J. Transl. Med 13, 138 (2015).

21. Lee, S. et al. Transcription factor NFAT5 promotes migration and invasion of rheumatoid synoviocytes via coagulation factor III and CCL2. J. Immunol. 201, 359-370 (2018).

22. Guo, K. \& Jin, F. NFAT5 promotes proliferation and migration of lung adenocarcinoma cells in part through regulating AQP5 expression. Biochem. Biophys. Res. Commun. 465, 644-649 (2015).

23. Li, X. et al. Mitochondria-translocated PGK1 functions as a protein kinase to coordinate glycolysis and the TCA cycle in tumorigenesis. Mol. Cell $\mathbf{6 1}$ 705-719 (2016).

24. Dong, W., Li, H. \& Wu, X. Rab11-FIP2 suppressed tumor growth via regulation of PGK1 ubiquitination in non-small cell lung cancer. Biochem Biophys. Res Commun. 508, 60-65 (2019).

25. Qian, X., Li, X. \& Lu, Z. Protein kinase activity of the glycolytic enzyme PGK1 regulates autophagy to promote tumorigenesis. Autophagy 13, 1246-1247 (2017).

26. Chang, H. H. et al. Metformin decreases the incidence of pancreatic ductal adenocarcinoma promoted by diet-induced obesity in the conditional KrasG12D mouse model. Sci. Rep. 8, 5899 (2018). 